\title{
Periodic-by-Nilpotent Linear Groups
}

\author{
B. A. F. WEHRFRITZ
}

ABstRACT - Let $G$ be a linear group of (finite) degree $n$ and characteristic $p \geq 0$. Suppose that for every infinite subset $X$ of $G$ there exist distinct elements $x$ and $y$ of $X$ with $\left\langle x, x^{y}\right\rangle$ periodic-by-nilpotent. Then $G$ has a periodic normal subgroup $T$ such that if $p>0$ then $G / T$ is torsion-free abelian and if $p=0$ then $G / T$ is torsion-free nilpotent of class at most $\max \{1, n-1\}$ and is isomorphic to a linear group of degree $n$ and characteristic zero. We also discuss the structure of periodic-by-nilpotent linear groups.

In [4] Rouabhi and Trabelsi prove that if $G$ is a finitely generated soluble-by-finite group such that for every infinite subset $X$ of $G$ there exist distinct elements $x$ and $y$ of $X$ with $\left\langle x, x^{y}\right\rangle$ periodic-by-nilpotent, then $G$ is periodic-by-nilpotent, work that ultimately was prompted by very much earlier work of B.H.Neumann, see [4]. Throughout for any positive integer $n$ we set $n^{\prime}=\max \{1, n-1\}$. Here we prove the following.

THEOREM. Let $G$ be a linear group of (finite) degree $n$ and characteristic $p \geq 0$. Suppose that for every infinite subset $X$ of $G$ there exist distinct elements $x$ and $y$ of $X$ with $\left\langle x, x^{y}\right\rangle$ periodic-by-nilpotent. Then $G$ is periodic-by-nilpotent. Further $G$ has a periodic normal subgroup $T$ such that if $p>0$ then $G / T$ is torsion-free abelian and if $p=0$ then $G / T$ is torsion-free nilpotent of class at most $n^{\prime}=\max \{1, n-1\}$ and is isomorphic to a linear group of degree $n$ and characteristic zero.

If $T$ is a periodic normal subgroup of some linear group $G$ of degree $n$ and characteristic zero, then $G / T$ is always isomorphic to a linear group of

Indirizzo dell'A.: School of Mathematical Sciences, Queen Mary University of London, Mile End Road, London E1 4NS, England.

E-mail: b.a.f.wehrfritz@qmul.ac.uk

MSC: 20H20, 20F45. 
characteristic zero (see [9]), but not necessarily of degree $n$, so the above situation is unusual. As a simple example, $\operatorname{SL}(2,5)$ has a faithful representation of degree 2 over the complex numbers (indeed over $\boldsymbol{Q}(\sqrt{5}, \sqrt[5]{1})$ ), but the least degree of a faithful representation in characteristic zero of its image $\operatorname{PSL}(2,5) \cong \operatorname{Alt}(5)$ is 3 .

Not every torsion-free nilpotent group is isomorphic to a linear group; for example, a direct product $D$ of infinitely many copies of the full unitriangular group $\operatorname{Tr}_{1}(3, \boldsymbol{Z})$ over the integers $\boldsymbol{Z}$ is torsion-free nilpotent of class 2 , but is not isomorphic to any linear group of any characteristic. Every torsion-free abelian group is isomorphic to a linear group of arbitrary characteristic ([6] 2.2) and any finitely generated torsion-free nilpotent group is isomorphic to a unipotent linear group over the integers (see [6] Page 23), so the counter example $D$ above is about as small as one can get.

For any group $G$ denote its hypercentre by $\zeta(G)$ and the $i$-th terms of its upper and lower central series by $\zeta_{i}(G)$ and $\gamma^{i} G$ respectively (where $\zeta_{0}(G)=\langle 1\rangle$ and $\left.\gamma^{1} G=G\right)$. Let $G$ be a group. If $\gamma^{m+1} G$ is finite, then $G / \zeta_{2 m}(G)$ is finite (if $G$ is finitely generated even $G / \zeta_{m}(G)$ is finite) and if $G / \zeta_{m}(G)$ is finite, then $\gamma^{m+1} G$ is finite, see [3] 4.25, 4.24 and 4.21, Corollary 2. Something similar happens for periodic-by-nilpotent linear groups.

Proposition. Let $G$ be a linear group of degree $n$ and characteristic $p \geq 0$.

a) Suppose that $\gamma^{m+1} G$ is periodic for some integer $m \geq 0$ and that $O_{p}(G)=\langle 1\rangle$ if $p>0$. Then $G / \zeta_{m}(G)$ (and $\gamma^{m+1} G$ ) are locally finite.

b) If $G / \zeta(G)$ is periodic, then $\gamma^{n^{\prime}+1} G$ and $G / \zeta_{n^{\prime}}(G)$ are locally finite.

c) If $G / \zeta_{m}(G)$ is periodic for some integer $m \geq 0$, then $\gamma^{m+1} G$ and $G / \zeta_{m}(G)$ are locally finite.

Of course Part $c$ ) only adds to Part $b$ ) in the Proposition for $m<n^{\prime}$ and Part a) for $m>n^{\prime}$ adds nothing to the case $m=n^{\prime}$ by the Theorem. Perhaps Part $b$ ) is a slight surprise, since if $G / \zeta(G)$ is finite there is no need for any $\gamma^{i} G$ to be finite, even if $G$ is also linear (consider the infinite locally dihedral 2 -group). If $G$ is any group with $G / \zeta_{m}(G)$ locally finite, then $\gamma^{m+1} G$ is easily seen to be locally finite.

If $G$ is the wreath product of a cyclic group of prime order $p$ by an infinite cyclic group, then $G$ is isomorphic to a triangular linear group of degree 2 and characteristic $p$ with $\gamma^{2} G$ periodic (even elementary abelian) and yet $\zeta(G)=\langle 1\rangle$. Thus the extra hypothesis if $p>0$ in Part a) cannot be 
removed. There is no obvious analogue to Part b) in the context of Part a); if $G$ is a non-cyclic free group, then $G$ is isomorphic to a linear group of degree 2 in any characteristic and yet $\gamma^{\omega} G=\langle 1\rangle=\zeta(G)$. Note that there exist hypercentral linear groups of infinite central height, even periodic ones, see [6] 8.3, so for example in Part $b$ ) there is no need for $\zeta(G)$ and $\zeta_{n^{\prime}}(G)$ to be equal.

Let $G$ be any group. Denote its unique maximal periodic normal subgroup by $\tau(G)$ and its unique maximal normal $\pi$-subgroup for $\pi$ some set of primes by $O_{\pi}(G)$. If $G$ is linear, $G^{0}$ denotes its connected component containing the identity (relative to the Zariski topology).

Proof of the Theorem. To begin with, note that if $G$ is a torsion-free, locally nilpotent group with a normal subgroup $H$ such that $G / H$ is periodic and such that $H$ is nilpotent of class $c$, then $G$ is nilpotent of class $c$. This can be derived either from isolator theory, see [2] 2.3.9, or from the Zariski topology using [5]. 5.11 and Point 3 on Page 23.

Suppose $G$ is a subgroup of $G L(n, F)$, where $F$ is an algebraically closed field of characteristic $p \geq 0$. If $G$ is not soluble-by-(locally finite), then $G$ contains a free subgroup on an infinite set $X$ by Tits' Theorem, see [6] 10.17. Then $\left\langle x, x^{y}\right\rangle$ is free of rank 2 for every pair of distinct elements $x$ and $y$ of $X$. Consequently $G$ is soluble-by-(locally finite). By Rouabhi \& Trabelsi's theorem, see [4], the group $G$ is locally (periodic-by-nilpotent) and hence is locally (periodic-by-(torsion-free nilpotent)). Therefore $G$ is periodic-by-(torsion-free, locally nilpotent).

Set $T=\tau(G)$ and suppose $p$ is positive. Clearly $O_{p}(G) \leq T$, so $G / T$ is isomorphic to a torsion-free, locally nilpotent, linear group of characteristic $p$ by Corollary 1 of [9]. Then $G / T$ is also abelian-by-finite by [6] 3.6 and consequently $G / T$ is abelian by the remark at the beginning of this proof. This settles the positive characteristic case.

From now on assume that $p=0$. Set $C=C_{G}(T)$. Then $G / C T$ is finite by [5] 5.1.6. Also $C$ is locally nilpotent, so $C$ has a Jordan decomposition

$$
C \leq C_{u} \times C_{d}=C_{u} C=C C_{d}=G L(n, F),
$$

see [6] Chapter 7, especially 7.14 and 7.13 (recall $F$ here is algebraically closed). Here $C_{u}$ is unipotent, torsion-free and nilpotent of class less than $n$. Set $P=\tau\left(C_{d}\right)$. Then $C_{d} / P$ is torsion-free, locally nilpotent and abelian-byfinite ([6] $7.7 \& 3.5)$. Therefore $C_{d} / P$ is abelian. Consequently $P=\tau\left(C_{u} C_{d}\right)$, $C \cap T=C \cap P$ and $C T / T \cong C /(C \cap P)$ is nilpotent of class at most $\max \{n-1,1\}=n^{\prime}$. But then $G / T$ is torsion-free, locally nilpotent and has a nilpotent subgroup $C T / T$ of finite index and class at most $n^{\prime}$. Therefore $G$ 
is torsion-free and nilpotent of class at most $n^{\prime}$, again by our remark at the beginning.

Finally $G / T$ is isomorphic to a linear group over $F$ of $n$-bounded degree by the theorem of [9], but we need to ensure it is actually isomorphic to a linear group of degree $n$ and characteristic zero. If $n=1$, then $G$ is abelian and clearly $G / T$ embeds into $F^{*}=G L(1, F)$, since $F^{*}$ is divisible and splits over $\tau\left(F^{*}\right)$. Suppose $n>1$. If $K$ is an extension field of $F$, then the centre of the unitriangular group $\operatorname{Tr}_{1}(n, K)$ is isomorphic to the additive group of $K$ and hence is equal to $Z \times R$ for $Z$ the centre of $\operatorname{Tr}_{1}(n, F)$ and $R$ a direct sum of copies of the additive group of the rationals. Further $C_{u}$ is isomorphic to a subgroup of $\operatorname{Tr}_{1}(n, F)$ and $C_{d} / P$ is embeddable in $R$ for a suitably large $K$. In which case $C_{u} C_{d} / P$ is isomorphic to a subgroup of $\operatorname{Tr}_{1}(n, K)$ and hence so too is $C T / T \cong C P / P \leq C_{u} C_{d} / P$. Now $\operatorname{Tr}_{1}(n, K)$ is torsion-free, nilpotent and divisible. Thus $\operatorname{Tr}_{1}(n, K)$ contains a divisible completion $D$ of $C T / T$. Since $G / T$ is torsion-free, nilpotent and of finite index over $C T / T$, so $D$ contains a copy of $G / T$. (See [2] Chap. 2, especially 2.1.1, for divisible completions of nilpotent groups.). Therefore $G / T$ is isomorphic to a linear group of degree $n$ and characteristic zero. The proof is complete.

As an example of an application of this theorem, we have the following.

COROLlaRY. Let $G$ be a soluble-by-finite group with finite Hirsch number. Suppose that for every infinite subset $X$ of $G$ there exist distinct elements $x$ and $y$ of $X$ with $\left\langle x, x^{y}\right\rangle$ periodic-by-nilpotent. Then $G$ is periodic-by-nilpotent.

Proof. For if $T=\tau(G)$, then $G / T$ has a torsion-free soluble normal subgroup of finite rank and index. Then $G / T$ is isomorphic to a linear group over the rationals (e.g. [7] 1.2) and applying the theorem to $G / T$ yields the corollary. Alternatively it follows from [4] as follows. By [4] the group $G$ is locally periodic-by-nilpotent, so $G$ is periodic-by-(torsion-free and locally nilpotent of finite rank). Consequently $G$ is periodic-by-nilpotent by a theorem of Mal'cev ([3] 6.36).

The Proposition follows at once from the following three lemmas.

LEMma 1. Let $G$ be a linear group of degree $n$ and characteristic $p \geq 0$ such that $O_{p}(G)=\langle 1\rangle$ if $p>0$. If there exists an integer $m \geq 0$ such that $\gamma^{m+1} G$ is periodic, then $G / \zeta_{m}(G)$ is locally finite.

Note that here $\gamma^{m+1} G$ is locally finite by [6] 4.9. 
Proof. Let $X$ be a finitely generated subgroup of $G$. Suppose first that $p=0$. Then $\gamma^{m+1} X$ is finite by [6] 4.8 and therefore $X / \zeta_{m}(X)$ is finite by [3] 4.24. Also $\zeta_{m}(X)$ is closed in $X$ by [6] 5.10 , so $X^{0} \leq \zeta_{m}(X)$. If $Y$ is a finitely generated subgroup of $G$ containing $X$, then

$$
X^{0} \leq X \cap Y^{0} \leq \zeta_{m}(Y)
$$

Thus $X^{0} \leq \zeta_{m}(G)$. Set $G^{*}=\bigcup_{X} X^{0}$. Then $G^{*}$ is a normal subgroup of $G$ with $G / G^{*}$ locally finite and $G^{*} \leq \zeta_{m}(G)$. The case $p=0$ follows.

Now assume that $p>0$. Here [6] 4.8 only yields that $\gamma^{m+1} X$ is a finite extension of a $p$-group. Define $Z_{i}(X)$ by

$$
Z_{i}(X) / O_{p}(X)=\zeta_{i}\left(X / O_{p}(X)\right) .
$$

Then $X / Z_{m}(X)$ is finite by [3] 4.24 and $O_{p}(X)$ and $Z_{m}(X)$ are closed in $X$, so $X^{0}=Z_{m}(X)$. Thus $X^{0} \leq \bigcap_{Y>X} X \cap Z_{m}(Y)$ and a simple localizing argument (cf. the previous paragraph) yields that $\left[G^{*},{ }_{m} G\right]$ is a $p$-group. Clearly it is normal in $G$ and $O_{p}(G)=\langle 1\rangle$. Therefore $G^{*} \leq \zeta_{m}(G)$ and the lemma follows.

LEMMA 2. Let $G$ be a linear group of degree n with $G / \zeta_{m}(G)$ periodic for some integer $m \geq 0$. Then $\gamma^{m+1} G$ and $G / \zeta_{m}(G)$ are locally finite.

Proof. Now $\zeta_{m}(G)$ is closed in $G$, so $G / \zeta_{m}(G)$ is isomorphic to a periodic linear group ([6] 6.4) and so is locally finite ([6] 4.9). Then [3] 4.21, Corollary 2 , yields that $\gamma^{m+1} X$ is finite for every finitely generated subgroup $X$ of G. Consequently $\gamma^{m+1} G$, which equals $\bigcup_{X} \gamma^{m+1} X$, is locally finite.

LEMMA 3. Let $G$ be a linear group of degree $n$ and characteristic $p \geq 0$ such that $G / \zeta(G)$ is periodic. Then $\gamma^{n^{\prime}+1} G$ and $G / \zeta_{n^{\prime}}(G)$ are locally finite.

Proof. We may assume that the ground field $F$ of $G$ is algebraically closed. If $g \in G L(n, F)$, let $g=g_{u} g_{d}=g_{d} g_{u}$ be its Jordan decomposition (see [6] Chapter 7). Set

$$
K=\left\langle g_{u}, g_{d}: g \in \zeta(G)\right\rangle \leq G L(n, F)
$$

Then $K=\zeta(G K), G \cap K=\zeta(G)$ and $K=K_{u} \times K_{d}$, where $K_{u}$ is unipotent and $K_{d}$ is a d-subgroup, see [6] 7.17, 7.14 and 7.13. Also $G K / K \cong G / \zeta(G)$, which is periodic.

By the theorem of [8] we have $K_{u} \leq \zeta_{n^{\prime}}(G K)$. Set $D=\left(K_{d}\right)^{0}$. Then $D$ is a diagonalizable normal subgroup of $G K$ by [6] 7.7 and 5.8. Let $\pi$ denote the finite set of all primes not exceeding $n$. Then $O_{\pi}(D)$ has finite rank (at most $n)$, so $D$ splits over $O_{\pi}(D)$ by [1] 21.2 and 27.5 , say $D=O_{\pi}(D) \times E$. Also 
$G K / C_{G K}(D)$ is a finite $\pi$-group by [6] 1.12. Then $H=\bigcap_{g \in G K} E^{g}$ is a normal subgroup of $G K$ with $O_{\pi}(H)=\langle 1\rangle$ and $D / H$ a periodic $\pi$-group. Also $[H, G K]$ is a $\pi$-group by [6] 4.14. Therefore $[H, G K]=\langle 1\rangle$. We have now shown that $K_{u} \times H=\cong \zeta_{n^{\prime}}(G K)$. Since $G K / K$ and $D / H$ are periodic and $K_{d} / D$ is finite, so $G K / \zeta_{n^{\prime}}(G K)$ is periodic. It follows that $G / \zeta_{n^{\prime}}(G)$ is periodic. Finally $\gamma^{n^{\prime}+1} G$ and $G / \zeta_{n^{\prime}}(G)$ are locally finite by Lemma 2 .

\section{REFERENCES}

[1] L. Fuchs, Infinite Abelian Groups Vol. 1, Academic Press, New York 1970.

[2] J. C. Lennox - D. J. S. RoBinson, The Theory of Infinite Soluble Groups, Clarendon Press, Oxford 2004.

[3] D. J. S. Robinson, Finiteness Conditions and Generalized Soluble Groups (2 vols.), Springer-Verlag, Berlin 1972.

[4] T. Rouabhi - N. Trabelsi, A note on torsion-by-nilpotent groups, Rend. Sem. Mat. Univ. Padova, 117 (2007), pp. 175-179.

[5] M. Shirvani - B. A. F. Wehrfritz, Skew Linear Groups, Cambridge Univ. Press, Cambridge 1986.

[6] B. A. F. Wehrfritz, Infinite Linear Groups, Springer-Verlag, Berlin 1973.

[7] B. A .F. WeHrFRITZ, On the holomorphs of soluble groups of finite rank, J. Pure \& Appl. Algebra, 4 (1974), pp. 55-69.

[8] B. A. F. WEHRFRITZ, Hypercentral unipotent subgroups of linear groups, Bull. London Math. Soc. 10 (1978), pp. 310-313.

[9] B. A. F. Wehrfritz, Periodic normal subgroups of linear groups, Arch. Math. (Basel), 71 (1998), pp. 169-172.

Manoscritto pervenuto in redazione il 16 ottobre 2009. 